International Journal of Pure and Applied Mathematics

Volume 98 No. $4 \quad 2015,485-489$

ISSN: 1311-8080 (printed version); ISSN: 1314-3395 (on-line version)

url: http://www.ijpam.eu

doi: http://dx.doi.org/10.12732/ijpam.v98i4.7

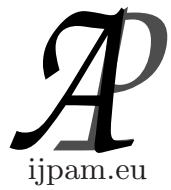

\title{
BEST CO-APPROXIMATION IN CONE METRIC SPACES
}

\author{
T. Sistani ${ }^{1}$, M. Abrishami-Moghaddam ${ }^{2} \S$ \\ ${ }^{1}$ Department of Mathematics \\ Kerman Branch \\ Islamic Azad University \\ Kerman, IRAN \\ ${ }^{2}$ Department of Mathematics \\ Birjand Branch \\ Islamic Azad University \\ Birjand, IRAN
}

AMS Subject Classification: 41A65

Key Words: cone metric spaces, best co-approximation, co-Chebyshev subset

\section{Introduction and Preliminaries}

Another kind of Approximation, called best co-approximation, was introduced by Franchettei and Furi in 1972 [1]. Some results on best co-approximation theory in metric and linear normed spaces have been obtained by P.L. Papini, I. Singer, T.D. Narang, and others (see [1], [4], [5], [6]). Cone metric spaces was introduced by Huang and Zhang in 2007 [2]; where the set of real numbers is replaced by an ordered Banach space. They introduced the basic definition and discuss some properties of convergence of sequences in cone metric spaces. Recently, Sh. Rezapour has a research work on best approximation in cone metric spaces [7]. In this paper we want to investigate the concept of best co-approximation in cone metric spaces.

Received: September 8, 2014

(c) 2015 Academic Publications, Ltd. url: www.acadpubl.eu

${ }^{\S}$ Correspondence author 
Let $E$ be a real Banach space and $P$ a subset of $E . P$ is called a cone if:

(i) $P$ is closed, non-empty and $P \neq\{0\}$,

(ii) $a x+b y \in P$ for all $x, y \in P$ and all non-negative real numbers $a, b$,

(iii) $P \cap(-P)=\{0\}$.

For a given cone $P \subseteq E$, we can define a partial ordering $\leq_{P}$ with respect to $P$ by $x \leq_{P} y$ if and only if $y-x \in P$. In what follows we omit the index $P$ and write everywhere $\leq$ instead of $\leq_{P} . x<y$ will stand for $x \leq y$ and $x \neq y$, while $x \ll y$ will stand for $y-x \in \operatorname{int} P$, where int $P$ denotes the interior of $P$.

In the following we always suppose that $E$ is a Banach space, $P$ is a cone in $E$ with int $P \neq \emptyset$ and $\leq$ is partial ordering with respect to $P$.

Definition 1. [2] Let $X$ be a non-empty set. Suppose the mapping $d: X \times X \rightarrow E$ satisfies: (d1) $0 \leq d(x, y)$ for all $x, y \in X$ and $d(x, y)=0$ if and only if $x=y$,

(d2) $d(x, y)=d(y, x)$ for all $x, y \in X$,

(d3) $d(x, y) \leq d(x, z)+d(z, y)$ for all $x, y, z \in X$.

Then $d$ is called a cone metric on $X$, and $(X, d)$ is called a cone metric space.

This definition is more general than that of a metric space.

Example 2. [2] Let $E=\mathbb{R}^{2}, P=\{(x, y) \in E: x, y \geq 0\} \subset \mathbb{R}^{2}$, $X=\mathbb{R}^{2}$ and suppose that $d_{i}: X \times X \rightarrow E, i=1,2$ where defined by $d_{1}(x, y)=$ $(|x-y|, \alpha|x-y|)$ and $d_{2}(x, y)=d_{2}\left(\left(x_{1}, x_{2}\right),\left(y_{1}, y_{2}\right)\right)=\left(\left|x_{1}-y_{1}\right|+\mid x_{2}-\right.$ $\left.y_{2} \mid, \alpha \max \left(\left|x_{1}-y_{1}\right|,\left|x_{2}-y_{2}\right|\right)\right)$, where $\alpha \geq 0$ is a constant. Then $\left(X, d_{i}\right), i=$ 1,2 , are cone metric spaces.

Example 3. [7] Let $E=\ell^{1}, P=\left\{\left\{x_{n}\right\}_{n \in \mathbb{N}} \in E: x_{n} \geq 0\right.$, for all $\left.n\right\}$, $(X, \rho)$ a metric space and $d: X \times X \rightarrow E$ defined by $d(x, y)=\left\{\frac{\rho(x, y)}{2^{n}}\right\}_{n \in \mathbb{N}}$. Then $(X, d)$ is a cone metric space.

This example shows that the category of cone metric spaces is bigger than the metric spaces.

Definition 4. [2] Let $(X, d)$ be a cone metric space, $x \in X$ and $\left\{x_{n}\right\}_{n \in \mathbb{N}}$ a sequence in $X$. Then $\left\{x_{n}\right\}_{n \in \mathbb{N}}$ converges to $x$ whenever for every $c \in E$ with $0 \ll c$ there is a natural number $N$ such that $d\left(x_{n}, x\right) \ll c$ for all $n \in \mathbb{N}$. We denote this by $\lim _{n \rightarrow \infty} x_{n}=x$ or $x_{n} \rightarrow x$. 
Definition 5. [7] Let $(X, d)$ be a cone metric space and $B \subseteq X$. If every sequence in $B$ has a convergent subsequence to an element of $B$, then $B$ is called a sequentially compact subset of $X$.

Definition 6. Let $(X, d)$ be a cone metric space, $G$ a non-empty subset of $X$ and $x \in X$. We say that $g_{0} \in \mathrm{G}$ is a best co-approximation of $x$ whenever $d\left(g, g_{0}\right) \leq d(x, g)$ for all $g \in G$. Then, we denote the set of all best co-approximations of $x$ in $G$ by $R_{G}(x)$. We say that $G$ is a co-Chebyshev subset of $X$ if $R_{G}(x)$ is a singleton subset of $G$ for all $x \in X$. Also, we say that $G$ is a quasi co-Chebyshev subset of $X$ if $R_{G}(x)$ is sequentially compact subset of $X$ for all $x \in X$.

Definition 7. Let $(X, d)$ be a cone metric linear space i.e. $X$ is a real vector space and $(X, d)$ a cone metric space. Also let $G$ be a non-empty subset of $X$. We say that $G$ is a pseudo co-Chebyshev subset of $X$ if there is no $x \in X$ such that $R_{G}(x)$ contains infinitely many linearly independent elements.

Definition 8. Let $(X, d)$ be a cone metric linear space. An element $x \in X$ is said to be orthogonal to another element $y \in X$, and write $x \perp_{P} y$, if $d(x, 0) \leq d(x, \alpha y)$ or $d(x, \alpha y)-d(x, 0) \in P$ for all scalar $\alpha . x$ is said to be orthogonal to a subset $G$ of $X\left(x \perp_{P} G\right)$ if $x \perp_{P} y$ for all $y \in G$.

\section{Main Results}

Theorem 9. If $G$ is a linear subspace of a cone metric space $(X, d)$ and $g_{0} \in G$, then $g_{0} \in R_{G}(x)$ if $G \perp_{P}\left(x-g_{0}\right)$.

Proof. If $G \perp_{P}\left(x-g_{0}\right)$, then $d(g, 0) \leq d\left(g, \alpha\left(x-g_{0}\right)\right)$ for all $g \in G$ and all $\alpha$, since $G$ is linear subspace so $d\left(\alpha g_{0}, g\right) \leq d(\alpha x, g)$, when $\alpha=1$ we have $g_{0} \in R_{G}(x)$.

Corollary 10. Let $G$ be a linear subspace of cone metric linear space $(X, d)$, then $R_{G}(x)=\emptyset$ for every $x \in X \backslash G$, if there exists no $z \in X \backslash\{0\}$ such that $G \perp_{P} z$.

Theorem 11. Let $(X, d)$ be a cone metric space, $G$ a nonempty subset of $X, g_{0} \in G$ and $x \in X$. Then $g_{0} \in R_{G}(x)$ if and only if for every $g \in G$ there exist a function $f^{g}: X \rightarrow E$ such that $f^{g}\left(g_{0}\right)=d\left(g, g_{0}\right), f^{g}(x)-f^{g}\left(g_{0}\right) \in P$ and $d(x, g)-f^{g}(x) \in P$. 
Proof. First suppose that for each $g \in G$ there exists a function $f^{g}: X \rightarrow E$ such that $f^{g}\left(g_{0}\right)=d\left(g, g_{0}\right), f^{g}(x)-f^{g}\left(g_{0}\right) \in P$ and $d(x, g)-f^{g}(x) \in P$. Thus $d\left(g, g_{0}\right)=f^{g}\left(g_{0}\right) \leq f^{g}(x) \leq d(x, g)$ for all $g \in G$. Hence $g_{0} \in R_{G}(x)$.

For converse, for all $g \in G$, define $f^{g}: X \rightarrow E$ by $f^{g}(t)=d(g, t)$, then $f^{g}\left(g_{0}\right)=d\left(g, g_{0}\right)$ and $f^{g}(x)-f^{g}\left(g_{0}\right)=d(g, x)-d\left(g, g_{0}\right) \in P, d(x, g)-f^{g}(x)=$ $0 \in P$.

Corollary 12. Let $(X, d)$ be a cone metric space and $G \subseteq X$. Then, $G$ is a co-Chebyshev subset of $X$ if and only if for each $g \in G$, there don't exist $x \in X$, distinct elements $g_{1}, g_{2} \in G$ and a function $f^{g}: X \rightarrow E$ such that $f^{g}\left(g_{i}\right)=d\left(g, g_{i}\right), f^{g}(x)-f^{g}\left(g_{i}\right) \in P$ and $d(x, g)-f^{g}(x) \in P$ for $i=1,2$.

Theorem 13. Let $(X, d)$ be a cone metric space and $G \subseteq X$. Then:

(i) $G$ is quasi co-Chebyshev subset of $X$ if and only if for each $g \in G$, there don't exist $x \in X$, a sequence $\left\{g_{n}\right\}_{n \in \mathbb{N}}$ in $G$ without a convergent subsequence and a function $f^{g}: X \rightarrow E$ such that $f^{g}\left(g_{n}\right)=d\left(g, g_{n}\right), f^{g}(x)-f^{g}\left(g_{n}\right) \in P$ and $d(x, g)-f^{g}(x) \in P$ for all $n \in \mathbb{N}$.

(ii)Furthermore if $(X, d)$ is a cone metric linear space, then $G$ is pseudo co-Chebyshev subset of $X$ if and only if for each $g \in G$, there don't exist $x \in X$, infinitely many linearly independent elements $\left\{g_{n}\right\}_{n \in \mathbb{N}}$ in $G$ and a function $f^{g}: X \rightarrow E$ such that $f^{g}\left(g_{n}\right)=d\left(g, g_{n}\right), f^{g}(x)-f^{g}\left(g_{n}\right) \in P$ and $d(x, g)-f^{g}(x) \in P$ for all $n \in \mathbb{N}$.

Proof. (i) First suppose that for each $g \in G$, there exists $x \in X$, a sequence $\left\{g_{n}\right\}_{n \in \mathbb{N}}$ in $G$ without a convergent subsequence and a function $f^{g}: X \rightarrow E$ such that $f^{g}\left(g_{n}\right)=d\left(g, g_{n}\right), f^{g}(x)-f^{g}\left(g_{n}\right) \in P$ and $d(x, g)-f^{g}(x) \in P$ for all $n \in \mathbb{N}$. Then by theorem 2.3, $g_{n} \in R_{G}(x)$ for all $n \in \mathbb{N}$. It follows that $R_{G}(x)$ is not sequentially compact. For converse, suppose that $G$ is not quasi co-Chebyshev subset of $X$. Then there exist $x \in X$ and a sequence $\left\{g_{n}\right\}_{n \in \mathbb{N}}$ in $R_{G}(x)$ without a convergent subsequence. By theorem 2.3, for each $g \in G$ there exist $f^{g}: X \rightarrow E$ such that $f^{g}\left(g_{n}\right)=d\left(g, g_{n}\right), f^{g}(x)-f^{g}\left(g_{n}\right) \in P$ and $d(x, g)-f^{g}(x) \in P$ for all $n \in \mathbb{N}$.

(ii) Proof is similar to (i).

\section{References}

[1] C. Franchetti, M. Furi, Some characteristic properties of real Hilbert spaces, Rev. Roumaine Math. Pures Appl. 17 (1972), 1045-1048. 
[2] Huang Long-Guang, Zhang Xian, Cone metric spaces and fixed point theorems of contractive mappings, J. Math. Anal. Appl., 332 (2007), 1468-1476.

[3] H. Mazaheri, S.M.S. Modarres, Some results concerning proximinality and coproximinality, Nonlinear Anal. 62 (6) (2005), 1123-1126.

[4] T. D. Narang, S. P. Singh, Best coapproximation in metric linear spaces, Tamkang. J. Math., 30 (1999), No.4, 243-253.

[5] P. L. Papini and I. Singer, Best coapproximation in normed linear spaces, Monat. fr Math, 88 (1979), 27-44.

[6] G. S. Rao, R. Saravanan, Characterization of best uniform coapproximation, Approx. Theory Appl. 15 (1) (1999), 23-37.

[7] Sh. Rezapour, Best approximation in cone metric spaces, Math. Moravica. 11 (2007), 85-88. 
